\title{
A Game Theoretic Study of Energy Efficient Cooperative Wireless Networks
}

\author{
Donald Richard Brown III and Fatemeh Fazel
}

\begin{abstract}
In wireless networks, it is well-known that intermediate nodes can be used as cooperative relays to reduce the transmission energy required to reliably deliver a message to an intended destination. When the network is under a central authority, energy allocations and cooperative pairings can be assigned to optimize the overall energy efficiency of the network. In networks with autonomous selfish nodes, however, nodes may not be willing to expend energy to relay messages for others. This problem has been previously addressed through the development of extrinsic incentive mechanisms, e.g., virtual currency, or the insertion of altruistic nodes in the network to enforce cooperative behavior. This paper considers the problem of how selfish nodes can decide on an efficient energy allocation and endogenously form cooperative partnerships in wireless networks without extrinsic incentive mechanisms or altruistic nodes. Using tools from both cooperative and non-cooperative game theory, the three main contributions of this paper are (i) the development of Pareto-efficient cooperative energy allocations that can be agreed upon by selfish nodes, based on axiomatic bargaining techniques, (ii) the development of necessary and sufficient conditions under which "natural" cooperation is possible in systems with fading and non-fading channels without extrinsic incentive mechanisms or altruistic nodes, and (iii) the development of techniques to endogenously form cooperative partnerships without central control. Numerical results with orthogonal amplify-and-forward cooperation are also provided to quantify the energy efficiency of a wireless network with sources selfishly allocating transmission/relaying energy and endogenously forming cooperative partnerships with respect to a network with centrally optimized energy allocations and pairing assignments.
\end{abstract}

Index Terms: Cooperative communication, game theory, wireless networks.

\section{INTRODUCTION}

Multihop or cooperative transmission is often used in wireless ad hoc networks to increase energy efficiency by allowing packets to be delivered over several short links [1]. One or more intermediate nodes between the source and destination can assist in the transmission by forwarding or relaying the packet along the route to the destination. Autonomous nodes acting in their own self-interest, however, may refuse to use their limited resources to forward packets for other nodes. This can lead to inefficient use of the network resources since messages may have to be retransmitted or re-routed through different paths to the destination node [2].

Manuscript received February 5, 2010; approved for publication by Sanghoon Lee, Division II Editor, March 31, 2010.

This work was supported by NSF award CCF-0447743.

The authors are with the Electrical and Computer Engineering Department, Worcester Polytechnic Institute, Worcester, MA 01609, email: \{drb, ffazel @ece.wpi.edu.
Several techniques have been proposed to encourage cooperation and improve the efficiency of wireless ad hoc networks with selfish autonomous nodes. A comprehensive study of these techniques can be found in [3]. One well-studied technique to encourage cooperation among selfish nodes is the development of extrinsic incentive mechanisms, e.g., virtual currency [4], [5], where nodes are reimbursed for cooperation. The idea of virtual currency is intuitively appealing in many scenarios, but the use of virtual currency has the potential for fraud and/or collusion as discussed in [6]. Another technique that can induce cooperation is the introduction of altruistic nodes into the network [7] that punish misbehaving nodes. While both of these techniques have been shown to encourage cooperation among selfish nodes, they both require some level of central authority in the network to perform accounting or to strategically insert altruistic nodes. They also implicitly assume that the near-term costs and benefits of cooperative behavior are one-sided, hence remuneration is necessary to enable cooperation. While this assumption is true in some cases, recent studies, e.g., [8], have shown that the benefits of cooperation can be two-sided and have considered the question of when "natural" cooperation is possible in large networks without any central authority. In [9], a two-player relaying game based on the orthogonal amplifyand-forward (OAF) cooperative transmission protocol [10] was analyzed in a non-cooperative game-theoretic framework and it was shown that natural cooperation without extrinsic incentive mechanisms or altruistic nodes can emerge under certain conditions on the channels. Two limitations of this work, however, are that it used centrally-controlled energy allocations and did not consider networks with more than two source nodes.

This paper considers the problem of how selfish nodes can locally decide on an efficient energy allocation and endogenously form cooperative partnerships in wireless networks with two or more source nodes and without any sort of extrinsic incentive mechanisms, altruistic nodes, or central authority. We first use bargaining tools from cooperative game theory to determine efficient energy allocations that can be locally computed and agreed to by a pair of selfish nodes. We then develop a repeated-game framework and employ tools from non-cooperative game theory to describe necessary and sufficient conditions under which natural cooperation between a pair of nodes is possible. To extend our results to networks with more than two source nodes, we then consider the question of how to endogenously form cooperative partnerships in general networks and propose the use of the "stable roommates" algorithm [11] to form partnerships that are stable with respect to unilateral or bilateral deviations. Finally, numerical results with OAF cooperation are provided to quantify the energy efficiency of a wireless network with sources selfishly allocating transmission/relaying energy and endogenously forming cooperative partnerships with respect to a net- 
work with centrally optimized energy allocations and pairing assignments.

Unlike the previous studies on this subject, the novelty of the approach in this paper is that the nodes in the network behave selfishly without any form of central authority, community enforcement, or extrinsic incentive mechanisms. Selfish autonomous nodes endogenously form cooperative partnerships, locally determine efficient energy allocations, and cooperate by relaying messages during transmission sessions with multiple frames. Throughout this paper, we assume that nodes exhibit rational individual choice behavior, meaning that each individual source node has a consistent preference relation over all possible energy allocations and partners, and always chooses the most preferred feasible alternative. We also assume that nodes can always refuse to cooperate if it is in their best interest to do so.

The rest of the paper is organized as follows. Section II introduces the system model used throughout the paper. In Section III, we present a two-player relaying game in stage game formulation, develop axiomatic bargaining solutions to determine an optimum energy allocation that two selfish players can agree upon, and then develop necessary and sufficient conditions under which selfish nodes will cooperate and not defect under a repeated-game formulation for both fading and non-fading channels. Section IV extends these two-player results to networks with $K>2$ sources and describes a technique in which the sources can endogenously form stable cooperative pairings. Section $\mathrm{V}$ provides numerical energy efficiency examples based on OAF cooperative transmission and concluding remarks are made in Section VI.

\section{SYSTEM MODEL}

We consider an ad hoc wireless network with $L$ half-duplex nodes and a discrete model of time where nodes transmit information to other nodes in the network in transmission sessions of variable duration. The sets of source nodes and destination nodes in a given transmission session are denoted as $\mathcal{S}$ and $\mathcal{D}$, respectively, where $|\mathcal{S}|=|\mathcal{D}|=K \leq L / 2$ and $\mathcal{S} \cap \mathcal{D}=\emptyset$. Fig. 1 shows such a network for the case when $L=4$ and $K=2$. The destination node for source node $i \in \mathcal{S}$ is denoted as $d_{i} \in \mathcal{D}$. It is assumed that the number of nodes and/or the amount of offered network traffic is sufficiently large such that, in any given transmission session, $K \geq 2$ source nodes wish to transmit independent information to distinct destination nodes in the network. The channel $h_{i j}[n]$ between node $i \in \mathcal{S}$ and node $j \in \mathcal{D} \cup \mathcal{S} \backslash i$ in frame $n$ is assumed to be frequency non-selective. The squared channel magnitude between node $i$ and $j$ in frame $n$, normalized with respect to the power of the additive white Gaussian noise (AWGN) in the channel, is denoted as $H_{i j}[n]$.

In each transmission session, the $K$ source nodes involved in the transmission session take turns transmitting using timedivision multiple access (TDMA). A transmission session is composed of $N \geq 1$ frames and each frame is composed of $2 K$ timeslots as shown in Fig. 2 for the case when $K=2$. In the first $K$ timeslots of each frame, each source node transmits a packet to its destination. Due to the undirected nature of wireless

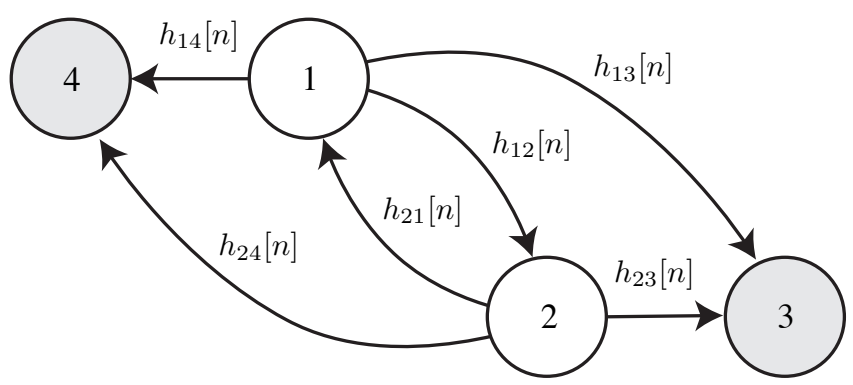

Fig. 1. An ad hoc wireless network with $L=4$ nodes. The two source nodes, shown in white and denoted as $\mathcal{S}=\{1,2\}$, wish to communicate independent information to the two destination nodes, shown in gray and denoted as $\mathcal{D}=\left\{d_{1}, d_{2}\right\}=\{3,4\}$.

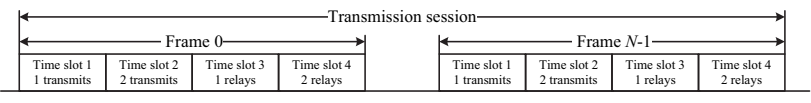

Fig. 2. A transmission session composed of $N$ frames with $\mathcal{S}=\{1,2\}$.

transmission, the TDMA transmissions in the first $K$ timeslots are also overheard by the other source nodes in the transmission session. The signal received by node $j$ from node $i$ in frame $n$ is given as

$$
y_{i j}[n]=\sqrt{H_{i j}[n]} x_{i}[n]+u_{i j}[n]
$$

for all $i \in \mathcal{S}$ and all $j \in \mathcal{D} \cup \mathcal{S} \backslash i$ in the current transmission session where $x_{i}[n]$ is the packet transmitted by source node $i$ in frame $n$ and $u_{i j}[n]$ is zero-mean unit-variance AWGN. In the remaining $K$ timeslots of the frame, each source node can potentially help one other source node by relaying a packet to its intended destination. When source node $j \in \mathcal{S} \backslash i$ elects to relay a packet for source node $i$, it transmits a function of the observation received from source node $i$ in the first half of the frame. Destination node $d_{i} \in \mathcal{D}$ receives

$$
z_{j d_{i}}[n]=\sqrt{H_{j d_{i}}[n]} f\left(y_{i j}[n]\right)+v_{j d_{i}}[n]
$$

where the relaying function $f$ depends on the cooperative protocol [10] and $v_{j d_{i}}[n]$ is zero-mean unit-variance AWGN. All noise terms are assumed to be spatially and temporally white. Note that each destination $d_{i} \in \mathcal{D}$ always receives at least one observation in each frame, i.e., the direct transmission $y_{i d_{i}}[n]$, and may receive two observations if another source node elects to relay the packet from source node $i$. It is assumed that the normalized channel magnitudes $\boldsymbol{H}[n]=\left\{H_{i j}[n]\right\}$ are either fixed (non-fading), i.e., $\boldsymbol{H}[n] \equiv \boldsymbol{H}$, or quasi-static and fading in the sense that $\boldsymbol{H}[n]$ is constant over the duration of each frame, but are independent and identically distributed (i.i.d.) in different frames of the transmission session. The current channel state is assumed to be known by the $K$ source nodes involved in the current transmission session. Channel phases are only assumed to be known at the respective receivers.

\section{TWO-PLAYER RELAYING GAME}

This section considers the scenario shown in Figs. 1 and 2 when there are two source nodes, denoted as $\mathcal{S}=\{1,2\}$, that 
wish to communicate independent information to two distinct destination nodes, denoted as $\mathcal{D}=\left\{d_{1}, d_{2}\right\}=\{3,4\}$. We extend the ideas developed in this section to the case with $K>2$ source nodes in Section IV.

\section{A. Stage Game Formulation}

A stage game is defined in terms of the players, available actions, and payoffs received by each player as a consequence of the actions for one frame of the current transmission session. The players in the game are the source nodes. In the first two timeslots of frame $n$, each source node transmits to its destination using transmit energy $\mathcal{E}_{1}[n]$ and $\mathcal{E}_{2}[n]$, respectively. If a source node does not request relaying from the other source node, i.e., it uses direct transmission to its destination, it will transmit with sufficient energy to satisfy a minimum qualityof-service (QoS) constraint, e.g., signal-to-noise ratio (SNR) or rate, at its destination based on the current channel state. For example, if the required at destination node $d_{i}$ is $\rho$, then source node $i$ will transmit with energy $\mathcal{E}_{i}[n]=\rho / H_{i d_{i}}[n]$ in frame $n$ when it uses direct transmission. We denote the required direct transmission energy for source node $i$ in frame $n$ as $\mathcal{E}_{i}^{d t}[n]$. If a source node requests relaying from the other source node in frame $n$, it will transmit with energy $0<\mathcal{E}_{i}[n]<\mathcal{E}_{i}^{d t}[n]$.

Referring to the timeslot schedule in Fig. 2, if node 2 has requested relaying, then node 1 must decide in timeslot 3 whether to fulfill this relaying request. Since both source nodes know the channel state, node 1 can determine the minimum relaying energy $\mathcal{E}_{1}^{r_{\min }}[n]$ required to ensure the QoS constraint is satisfied at destination node 4 [12]. Although node 1 can choose any non-negative relaying energy $\mathcal{E}_{1}^{r}[n]$, a selfish node will never rationally choose a relaying energy larger than the minimum required relaying energy since there is no benefit to either source node if a node expends excess relaying energy. If source node 1 transmits with relaying energy less than the minimum required relaying energy, then the packet will not be received at destination node 4 with the required QoS and node 2 will need to transmit with additional energy at the end of the frame to ensure the QoS constraint is satisfied. For these reasons, we assume that node 1 chooses from the discrete set of actions "do not relay" $\left(a_{1}[n]=\mathrm{DNR} \Leftrightarrow \mathcal{E}_{1}^{\mathrm{r}}[\mathrm{n}]=0\right)$ and "relay with minimum required relaying energy" $\left(a_{1}[n]=\mathrm{R} \Leftrightarrow \mathcal{E}_{1}^{\mathrm{r}}[\mathrm{n}]=\mathcal{E}_{1}^{\mathrm{r}_{\min }}[\mathrm{n}]\right)$ in frame $n$. If source node 1 chooses the action DNR when $\mathcal{E}_{1}^{r_{\min }}[n]>0$, then node 2 will transmit at the end of the frame with the remaining energy required to ensure the QoS constraint is satisfied at node 4 . Since the channel magnitudes are assumed to be constant over the duration of the frame, the total transmission energy expended by node 2 in this case will be the same as if node 2 had used direct transmission in timeslot 2 . If source node 1 chooses the action $\mathrm{R}$, the packet will be received by node 4 at the required QoS level without any additional transmission energy from node 2 .

In timeslot 4 , if node 1 has requested relaying, node 2 must also decide between the actions DNR and R. The situation is the same in this case as when node 1 relays for node 2 except that node 2 has the advantage of having just observed whether or not node 1 fulfilled its relaying request and can choose its action accordingly.

Since packets from both source nodes are always delivered

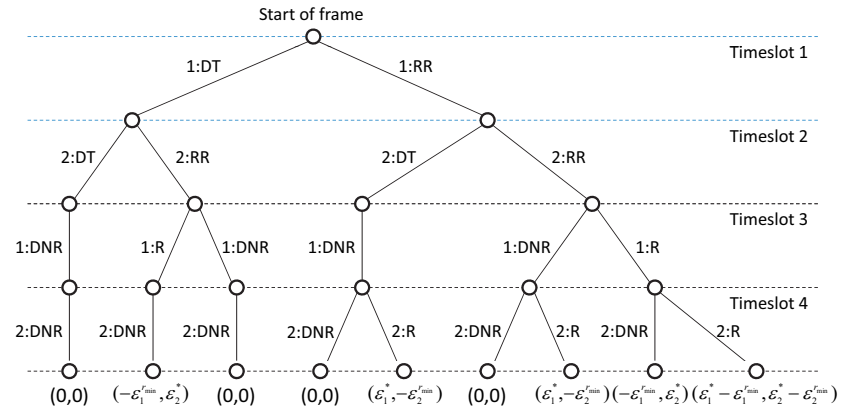

Fig. 3. Two-player relaying game in extensive form with source nodes $\mathcal{S}=\{1,2\}$. The actions DT, RR, R, and DNR correspond to "direct transmission (no relay request)," "request relay," "relay," and "do not relay," respectively. The pairs at the bottom of the tree correspond to the payoffs of nodes 1 and 2, respectively, at the end of the frame.

to each destination irrespective of whether relaying requests are fulfilled or not, we define the stage game payoff as the transmission energy saved in the current frame with respect to direct transmission. The payoff received by source node $i$ in frame $n$ is denoted as $\pi_{i}(\boldsymbol{a}[n], n)$ where

$$
\begin{aligned}
\boldsymbol{a}[n]= & \left(a_{1}[n], a_{2}[n]\right) \\
& \in\{(\mathrm{DNR}, \mathrm{DNR}),(\mathrm{DNR}, \mathrm{R}),(\mathrm{R}, \mathrm{DNR}),(\mathrm{R}, \mathrm{R})\}
\end{aligned}
$$

is the action profile of both players in frame $n$. Note that whenever $\boldsymbol{a}[n]=(\mathrm{DNR}, \mathrm{DNR})$, both source nodes receive a payoff of zero (both nodes transmitted with the same energy as direct transmission). If source node $i$ chooses $\mathrm{R}$ and node $j$ chooses $\mathrm{DNR}$, then node $i$ receives a payoff of $\pi_{i}(\boldsymbol{a}[n], n)=-\mathcal{E}_{i}^{r_{\mathrm{min}}}$ and node $j$ receives a payoff of $\pi_{j}(\boldsymbol{a}[n], n)=\mathcal{E}_{j}^{*}$ where

$$
\mathcal{E}_{j}^{*}[n]:=\mathcal{E}_{j}^{d t}[n]-\mathcal{E}_{j}[n]>0
$$

is defined as the energy saved by node $j$ with respect to direct transmission if node $j$ requests relaying and node $i \neq j$ fulfills the relaying request by relaying with sufficient energy to ensure the QoS constraint is satisfied at destination node $d_{j}$. Finally, if $\boldsymbol{a}[n]=(\mathrm{R}, \mathrm{R})$, the source nodes receive payoffs $\left(\pi_{1}(\boldsymbol{a}[n], n), \pi_{2}(\boldsymbol{a}[n], n)\right)=\left(\mathcal{E}_{1}^{*}-\mathcal{E}_{1}^{r_{\min }}, \mathcal{E}_{2}^{*}-\mathcal{E}_{2}^{r_{\text {min }}}\right)$. Fig. 3 summarizes the two-player relaying game in extensive form [13] and shows the payoffs received by each source node as a function of the actions chosen by the players in the current frame.

\section{B. Feasible Payoff Set and Pareto-Efficient Payoff Pairs}

Based on the two-player stage game formulation in the previous section, we note that both nodes transmit with energy $0<\mathcal{E}_{i}[n] \leq \mathcal{E}_{i}^{d t}[n]$ in the first two timeslots of the frame. This energy, along with the relaying actions of both nodes in the third and fourth timeslots of the frame, specifies the payoff pair that both source nodes receive in the frame. We denote the set of all non-negative feasible stage game payoffs as $\mathcal{U} \subset \mathbb{R}^{2}$ and a Pareto-efficient payoff pair as follows.

Definition 1: A payoff pair $\left(\pi_{1}, \pi_{2}\right) \in \mathcal{U}$ is Pareto-efficient if there exists no other payoff pair $\left(\pi_{1}^{\prime}, \pi_{2}^{\prime}\right) \in \mathcal{U}$ such that $\pi_{1}^{\prime}>\pi_{1}$ and $\pi_{2}^{\prime} \geq \pi_{2}$ or $\pi_{1}^{\prime} \geq \pi_{1}$ and $\pi_{2}^{\prime}>\pi_{2}$.

In other words, a payoff pair is Pareto-efficient if it is impossible for one source to improve its stage game payoff without 
reducing the payoff of the other source. We denote the set of all Pareto-efficient payoff pairs as $\hat{\mathcal{U}} \subseteq \mathcal{U}$.

The notion of natural cooperation, i.e., cooperation without extrinsic incentive mechanisms like virtual currency, is centered around the possibility that the set $\hat{\mathcal{U}} \backslash(0,0)$ is not empty. When this set is not empty, there exists at least one point where one source node does strictly better than direct transmission and the other node does no worse. When $\hat{\mathcal{U}} \backslash(0,0)=\emptyset$, it is clear that both nodes will use direct transmission, since one or both nodes will do worse than direct transmission otherwise. When $\hat{\mathcal{U}} \backslash(0,0) \neq \emptyset$, there remains the question of what payoff pair in the set $\hat{\mathcal{U}}$ the nodes should use. In a system with central control, one approach would be to minimize the total transmission/relaying energy by maximizing the total payoff $\pi_{1}+\pi_{2}$. Selfish nodes, however, may not agree to this division of the "surplus". The following section discusses how selfish source nodes can agree to a Pareto-efficient payoff pair using axiomatic bargaining tools from cooperative game theory.

\section{Axiomatic Bargaining for Cooperative Energy Allocation}

When the set $\hat{\mathcal{U}} \backslash(0,0)$ is not empty, selfish nodes will attempt to arrive at a unique mutually agreeable payoff pair (and, consequently, a unique energy allocation) through "bargaining". The bargaining problem is one of the paradigms of cooperative game theory in which a group of two or more participants are faced with a set of feasible outcomes, any of which can be the bargaining solution if agreed to unanimously. Our use of the term bargaining here is somewhat misleading in the sense that the nodes do not actually bargain by communicating offers and counteroffers to each other. Rather, since the channel state is known to both source nodes and each node knows how the other will bargain, each node can determine the bargaining solution locally without any additional communication. The technique of uniquely dividing a surplus among selfish players is commonly called "bargaining" in the cooperative game-theory literature, however, and we will use this term here for consistency.

Let us define the pair $(\mathcal{U}, \Delta)$ as the bargaining problem, where $\mathcal{U}$ is the set of all non-negative feasible stage game payoff pairs and $\Delta=(0,0)$ is the disagreement payoff. If both sources fail to reach an agreement, they use direct transmission to deliver their packets to their intended destinations and receive the disagreement payoff in the current stage game. Note that $\Delta$ is always in $\mathcal{U}$ because direct transmission is always feasible. It is assumed that $\mathcal{U}$ is a convex and closed set, bounded from above ${ }^{1}$. Given the definition of the bargaining problem and the set of Pareto-efficient payoff pairs $\hat{\mathcal{U}}$, an axiomatic bargaining solution is a function $B$, based on a set of "reasonable" axioms, that maps every $(\mathcal{U}, \Delta)$ to a unique member of $\hat{\mathcal{U}}$. Specifying these axioms serves to characterize the solution uniquely from among the set of Pareto-efficient points.

The most commonly used axiomatic bargaining solution is the Nash bargaining solution (NBS) which is based on four simple and well-accepted axioms and has been shown to have close connections to subgame-perfect equilibria in infinite horizon games [13]. These axioms can be briefly described as

\footnotetext{
${ }^{1}$ Sufficient conditions under which this assumption holds are provided in the Appendix.
}

1. (Pareto-efficiency) the bargaining solution $B(\mathcal{U}, \Delta)$ must be Pareto-efficient.

2. (Independence of linear transformations) if $T=a x+b$ with $a>0$, then the bargaining solution $B(T(\mathcal{U}), T(\Delta))=$ $T(B(\mathcal{U}, \Delta))$, i.e., the bargaining solution must be independent of the utility scales of the players.

3. (Symmetry) the bargaining solution $B(\mathcal{U}, \Delta)$ will give equal payoffs to both players if the set $\mathcal{U}$ is symmetric in the sense that $\left(u_{1}, u_{2}\right) \in \mathcal{U}$ implies $\left(u_{2}, u_{1}\right) \in \mathcal{U}$.

4. (Independence of irrelevant alternatives) if $\mathcal{V} \subseteq \mathcal{U}$ and $B(\mathcal{U}, \Delta) \in \mathcal{V}$, then $B(\mathcal{V}, \Delta)=B(\mathcal{U}, \Delta)$, i.e., the addition of irrelevant alternatives does not affect the bargaining solution.

Other axiomatic bargaining solutions based on different sets of axioms include the Raiffa Kalai-Smorodinsky (RBS) [14] and the modified Thomson bargaining solutions (MTBS). A unified view of all these axiomatic models is presented in [15].

The NBS, RBS, and MTBS bargaining solutions can all be expressed as

$$
B_{\beta}(\mathcal{U}, \Delta)=\arg \max _{\left(w_{1}, w_{2}\right) \in \hat{\mathcal{U}}} w_{1} w_{2}
$$

where $\beta$ is a scalar parameter specified by the bargaining solution ( $\beta=0$ for NBS, $\beta=1$ for RBS, and $\beta=-1$ for MTBS) and

$$
w_{i}:=\frac{\pi_{i}}{m_{i}}+\beta\left(1-\frac{\pi_{j}}{m_{j}}\right)
$$

is the preference function of source node $i$, with $j \in\{1,2\}$, $j \neq i$, and $m_{i}$ is the maximum stage game payoff of source $i$ over $\mathcal{U}$. The value of $\beta$ in the preference function implies a tradeoff between a player's own gain and the other player's losses, normalized by each player's maximum gain. When players use the NBS $(\beta=0)$, the bargaining solution is such that players only maximize their own payoff without consideration of the losses incurred by the other player. When players use the RBS $(\beta=1)$, the bargaining solution is such that each player's payoff is proportional to its maximum. Finally, when players use the MTBS ( $\beta=-1)$, each player has the same preference function and the bargaining solution is such that the sum of the normalized payoffs $\pi_{1} / m_{1}+\pi_{2} / m_{2}$ is maximized.

To illustrate the feasible payoff set, the Pareto-efficient subset, as well as the different bargaining solutions, Fig. 4 shows the positive quadrant of the feasible stage game payoffs as well as the NBS, RBS, and MTBS for a two-player relaying game using OAF cooperation with channel state $H_{12}=H_{21}=5$, $H_{13}=0.5, H_{14}=5, H_{23}=3$, and $H_{24}=0.9$ and an $\mathrm{SNR}=10 \mathrm{~dB}$ QoS constraint. The maximization in (1) is performed numerically for $\beta \in\{-1,0,1\}$ to obtain the three different bargaining solutions. The centrally controlled maximum total payoff (or, equivalently, minimum total energy), is also plotted for comparison.

\section{Stage Game Nash Equilibrium Analysis}

Given a bargaining solution in the two-player stage game such that $\mathcal{E}_{i}^{r_{\min }}>0$ for both source nodes, this section considers the question of whether selfish source nodes really will follow 


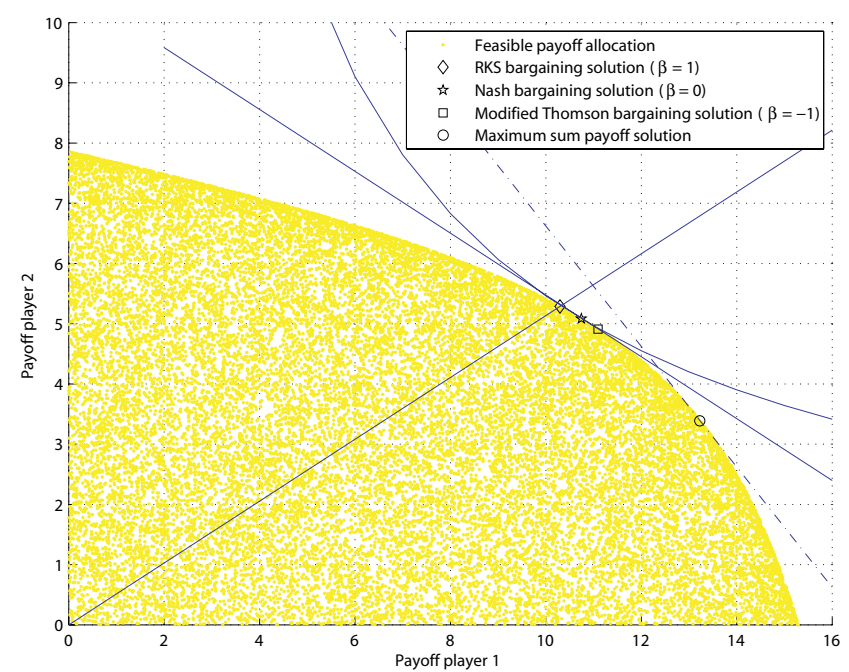

Fig. 4. Illustration of NBS, RBS, and MTBS two-player bargaining solutions over the set of feasible stage game payoffs, $\mathcal{U}$.

through on the agreement by looking at the consequences of defection in the context of non-cooperative game theory. Inspection of the payoff pairs in Fig. 3 shows that, when node 2 is requested to relay such that $\mathcal{E}_{2}^{r_{\text {min }}}>0$, node 2 will choose the action DNR because its payoff of choosing DNR is always better than choosing R, irrespective of node 1's actions. Knowing this, node 1 will also choose the action DNR for the same reasons. Since both source nodes know that any relay requests will always be rejected, both source nodes will choose to communicate with their respective destinations by direct transmission and receive the payoff pair $\left(\pi_{1}(\boldsymbol{a}[n], n), \pi_{2}(\boldsymbol{a}[n], n)\right)=(0,0)$.

To formalize this result, we briefly review the concept of a Nash Equilibrium (NE) [13]. In a $k$-player game, the action profile $\left(a_{1}^{*}, \cdots, a_{k}^{*}\right)$ is an NE if, for each player $i, a_{i}^{*}$ is player $i$ 's best response to $\boldsymbol{a}_{-i}^{*}$, where $\boldsymbol{a}_{-i}$ denotes the actions of all the players except player $i$. In frame $n$, this can be expressed as

$$
\pi_{i}\left(\left\{a_{i}^{*}[n], \boldsymbol{a}_{-i}^{*}[n]\right\}, n\right) \geq \pi_{i}\left(\left\{a_{i}[n], \boldsymbol{a}_{-i}^{*}[n]\right\}, n\right)
$$

for all $a_{i}$ in the set of available actions for player $i$ and where $\pi_{i}$ is the payoff function player $i$. Intuitively, if all of the players are choosing NE actions, no player can increase their payoff by unilaterally deviating from the NE action profile. It is not difficult to show that the only NE of the two-player relaying stage game is $\boldsymbol{a}[n]=(\mathrm{DNR}, \mathrm{DNR})$.

The dilemma in this result is that the bargaining solution developed in the previous section specifies an energy allocation such that both nodes would receive a payoff better than $(0,0)$ by accepting relay requests. In other words, if the channel state is such that both nodes could save energy through mutual cooperation, both nodes would do better by choosing $\boldsymbol{a}[n]=(\mathrm{R}, \mathrm{R})$ than $\boldsymbol{a}[n]=(\mathrm{DNR}, \mathrm{DNR})$. But selfish nodes must act rationally, and a simple non-cooperative game theoretical analysis of the extensive form of the stage game shows that there is only one NE action profile for selfish players: Mutual non-cooperation.In the following section, we extend this stage game analysis to a repeated game formulation and show that, unlike a single-stage game, a repeated game with uncertain ending, i.e., a transmis- sion session with an uncertain number of frames, can include a mutually cooperative NE for selfish players.

\section{E. Repeated-Game Nash Equilibrium Analysis}

Since each transmission session is composed of $N \geq 1$ frames, the stage game formulation developed in the prior section can be extended to a repeated-game model where the players interact over multiple stage games. If $N$ is known to both source nodes in the current transmission session, backward induction arguments can be used to show that both players will choose $\boldsymbol{a}[n]=(\mathrm{DNR}, \mathrm{DNR})$ in each stage game. To see this, first consider the last stage game. Since there is no possibility of gain from future cooperation, node 2 will rationally choose DNR to maximize its payoff. Knowing this, node 1 will also choose DNR for the same reasons. Since each node knows that the other node will choose DNR in the last stage game, they will also choose $\boldsymbol{a}[n]=(\mathrm{DNR}, \mathrm{DNR})$ in the second to last stage game, and so on, ensuring that the only rational strategy for both source nodes is to reject relay requests in all of the stage games [16, p.10].

Now consider the scenario when the number of frames in the current transmission session is not known by the source nodes. This scenario can occur, for example, in a cognitive radio network where the source nodes are secondary users and the end of the game occurs when the primary user becomes active. We consider the case when the transmission session continues after the current frame with fixed probability $\delta$, where $\delta$ is known to both source nodes. In this case, the number of frames $N$ is a geometrically distributed random variable with probability mass function $p_{N}(n)=(1-\delta) \delta^{n-1}$ for $n=1,2, \cdots$. Since the number of frames in the current transmission session is not known to the source nodes (and, as will be discussed in subsection III-E, the payoffs in future frames may also be unknown), both source nodes seek to maximize their expected total payoff in the transmission session. We define the expected total payoff of node $i$ as

$$
\Pi_{i}:=\mathrm{E}\left\{\sum_{n=0}^{N-1} \pi_{i}(\boldsymbol{a}[n], n)\right\}
$$

where $N$ is random and $\pi_{i}(\boldsymbol{a}[n], n)$ is random when the channels are fading. Under the assumption that the stage game payoffs are independent of $N$, the expected total payoff can be shown to be equivalent to a repeated game having an infinite number of stages with future payoffs discounted according to the expected duration of the game [16]. For player $i$, this can be expressed as

$$
\Pi_{i}=\sum_{n=0}^{\infty} \delta^{n} \mathrm{E}\left\{\pi_{i}(\boldsymbol{a}[n], n)\right\}
$$

where $\delta$ is called the discount factor and $\pi_{i}(\boldsymbol{a}[n], n)$ is the $i$ th player's payoff in frame $n$ given action profile $\boldsymbol{a}[n]$.

In repeated games, players use a strategy to specify their actions in each stage game as a function of the channel state, cooperative protocol, QoS constraint, and previous actions of the other players. We define a trigger strategy in the repeated twoplayer relaying game as follows: If the bargaining solution specifies $\mathcal{E}_{i}^{r_{\text {min }}}[n]>0$, player $i$ chooses the action $a_{i}[n]=\mathrm{R}$ unless the other player has previously chosen DNR when relaying was 
requested. If player $i$ chooses DNR when $\mathcal{E}_{i}^{r_{\min }}[n]>0$, then player $i$ is said to defect. If either player defects, the other player "triggers" punishment by choosing DNR in all future stage games (note that, since node 2 chooses its action after it observes the action of node 1 in the current stage game, node 2 will trigger punishment by playing DNR in the current stage game).

In our analysis of the repeated game scenario, the channel states in the current and previous frames are assumed to be known to both sources. When the channels are fading, the channel states in future frames are not known; only their distribution is known. The energy allocation

$$
\mathcal{E}[n]=\left\{\mathcal{E}_{1}[n], \mathcal{E}_{2}[n], \mathcal{E}_{1}^{r_{\min }}[n], \mathcal{E}_{2}^{r_{\min }}[n]\right\}
$$

is assumed to be dynamically determined via a bargaining solution in each frame $n=0,1, \cdots$ according to the known channel state, the cooperative protocol, and the QoS constraint. The following proposition establishes necessary and sufficient conditions under which the (TRIGGER,TRIGGER) strategy profile is an NE of the repeated two-player relaying game with uncertain ending in systems with quasi-static i.i.d. fading channels.

Proposition 1: In a system with quasi-static i.i.d. fading channels, the strategy profile (TRIGGER,TRIGGER) is an NE of the repeated two-player relaying game with uncertain ending if and only if $\mathcal{E}_{1}^{r_{\min }}[n] \leq \mathcal{E}_{1}^{*}[n]+\frac{\delta}{1-\delta} \overline{\mathcal{E}}_{1}$ and $\mathcal{E}_{2}^{r_{\min }}[n] \leq \frac{\delta}{1-\delta} \overline{\mathcal{E}}_{2}$ for all $n=0,1, \cdots$, where $\overline{\mathcal{E}}_{i}:=\mathrm{E}\left\{\mathcal{E}_{i}^{*}[n]-\mathcal{E}_{i}^{r_{\min }}[n]\right\}$.

Proof: In frame $n^{\prime}$, if both nodes have faithfully played and continue to play the strategy profile (TRIGGER,TRIGGER), they will receive an expected total payoff of

$$
\begin{aligned}
\Pi_{i} & =\sum_{n=0}^{n^{\prime}-1}\left(\mathcal{E}_{i}^{*}[n]-\mathcal{E}_{i}^{r_{\min }}[n]\right)+\left(\mathcal{E}_{i}^{*}\left[n^{\prime}\right]-\mathcal{E}_{i}^{r_{\min }}\left[n^{\prime}\right]\right) \\
& +\sum_{n=n^{\prime}+1}^{\infty} \delta^{n-n^{\prime}} \overline{\mathcal{E}}_{i} .
\end{aligned}
$$

The first and second terms in this expression correspond to the known total payoff of the previous frames and the known payoff of the current frame, respectively. The final term in this expression corresponds to the expected total payoff from mutual cooperation in future stage games where the i.i.d. channel state assumption has been used to remove the dependence of the mean on $n$.

If node 1 deviates from the TRIGGER strategy by defecting in stage game $n^{\prime}$, it will receive a total payoff of

$$
\Pi_{1}=\sum_{n=0}^{n^{\prime}-1}\left(\mathcal{E}_{1}^{*}[n]-\mathcal{E}_{1}^{r_{\min }}[n]\right)
$$

because node 2 will punish node 1 immediately for its defection in the current stage game. Note that this total expected payoff does not exceed the total expected payoff from faithfully playing the TRIGGER strategy when $\left(\mathcal{E}_{1}^{*}\left[n^{\prime}\right]-\mathcal{E}_{1}^{r_{\min }}\left[n^{\prime}\right]\right)+$ $\sum_{n=n^{\prime}+1}^{\infty} \delta^{n-n^{\prime}} \overline{\mathcal{E}}_{1} \geq 0$. Hence, node 1 has no incentive to deviate from the strategy profile (TRIGGER,TRIGGER) when $\mathcal{E}_{1}^{r_{\min }}\left[n^{\prime}\right] \leq \mathcal{E}_{1}^{*}\left[n^{\prime}\right]+\frac{\delta}{1-\delta} \overline{\mathcal{E}}_{1}$.
If node 2 deviates from the TRIGGER strategy by defecting in stage game $n^{\prime}$, it is punished by node 1 in the next stage game and receives a total expected payoff of

$$
\Pi_{2}=\sum_{n=0}^{n^{\prime}-1}\left(\mathcal{E}_{2}^{*}[n]-\mathcal{E}_{2}^{r_{\min }}[n]\right)+\mathcal{E}_{2}^{*}\left[n^{\prime}\right] .
$$

The second term here corresponds to the payoff received by node 2 in stage game $n^{\prime}$ when its packet is forwarded by node 1 but it does not reciprocate. This total expected payoff does not exceed the total expected payoff from faithfully playing the TRIGGER strategy when $\mathcal{E}_{2}^{r_{\min }}\left[n^{\prime}\right] \leq \frac{\delta}{1-\delta} \overline{\mathcal{E}}_{2}$.

Proposition 1 implies that, as long as both source nodes can find a bargaining solution that specifies an energy allocation such that the nodes are not requested to expend "too much" relaying energy in the current frame, then mutual cooperation (with the threat of punishment for defection) is an NE of the repeated two-player relaying game with uncertain ending. In each frame, each source node must check the NE conditions to determine if the bargaining solution implies an energy allocation that satisfies the NE criteria. If not, both sources use direct transmission in the current frame. Note that this action is not interpreted as defection since both sources have agreed to use direct transmission in this frame. We also note that the strategy profile (ALWAYS DEFECT, ALWAYS DEFECT) is also an NE of the repeated two-player relaying game with uncertain ending since neither player stands to gain from cooperation with an opponent that always defects.

It is worth mentioning here that the quantity $\overline{\mathcal{E}}_{i}:=$ $\mathrm{E}\left\{\mathcal{E}_{i}^{*}[n]-\mathcal{E}_{i}^{r_{\min }}[n]\right\}$ in Proposition 1 is implicitly defined in the sense that the expectation on the right hand side of this equality depends on $\overline{\mathcal{E}}_{1}$ and $\overline{\mathcal{E}}_{2}$. In the numerical results in Section $\mathrm{V}$, we calculate $\overline{\mathcal{E}}_{1}$ and $\overline{\mathcal{E}}_{2}$ numerically by first computing $N$ bargaining solutions based on $N$ quasi-static i.i.d. fading channel realizations and then computing the sample means $\frac{1}{N} \sum_{n=1}^{N}\left\{\mathcal{E}_{i}^{*}[n]-\mathcal{E}_{i}^{r_{\min }}[n]\right\}$ for $i=1,2$. These values are used as the first estimates of $\overline{\mathcal{E}}_{1}$ and $\overline{\mathcal{E}}_{2}$ to check whether the energy allocation specified by the bargaining solution in each frame satisfies the NE criteria. If any of the energy allocations fail to satisfy the NE criteria, the energy allocations in these frames are set to direct transmission. The sample means are then recomputed and this process is performed iteratively until the energy allocations in all of the frames satisfy the NE criteria based on the sample means.

As a special case of Proposition 1, we can also consider a system with non-fading channels where the channel state is the same over all of the frames in the transmission session, i.e., $\boldsymbol{H}[n] \equiv \boldsymbol{H}$ for all $n=0,1, \cdots$, and the sources use fixed energy allocations, i.e., $\mathcal{E}[n] \equiv\left\{\mathcal{E}_{1}, \mathcal{E}_{2}, \mathcal{E}_{1}^{r_{\min }}, \mathcal{E}_{2}^{r_{\min }}\right\}$ for all $n=0,1, \cdots$. The following corollary establishes necessary and sufficient conditions under which the (TRIGGER,TRIGGER) strategy profile is an NE of the repeated two-player relaying game with uncertain ending in systems with non-fading channels.

Corollary 1: In a system with non-fading channels, the strategy profile (TRIGGER,TRIGGER) is an NE of the repeated twoplayer relaying game with uncertain ending if and only if $\mathcal{E}_{1}^{r_{\min }} \leq \mathcal{E}_{1}^{*}$ and $\mathcal{E}_{2}^{r_{\min }} \leq \delta \mathcal{E}_{2}^{*}$. 
The proof of Corollary 1 follows from Proposition 1 by substituting $\overline{\mathcal{E}}_{i}=\mathcal{E}_{i}^{*}-\mathcal{E}_{i}^{r_{\min }}$ for $i \in\{1,2\}$. Unlike the case with fading channels, all of the future payoffs are known when the channels are non-fading; only the duration of the transmission session is unknown. Both source nodes have no incentive to defect when they expect the transmission session to be long enough such that they receive more long-term benefit from cooperation than short-term benefit from defection. The NE conditions are also simple to compute in this case.

In each frame $n=0,1, \cdots$, Proposition 1 (or Corollary 1 in systems with non-fading channels) identifies a set of feasible energy allocations under which selfish nodes will rationally choose mutual cooperation. If the bargaining solution from subsection III-C implies an energy allocation that is in this set, then selfish nodes will follow through on their agreement, relaying messages from each other and receiving stage game payoffs according to the bargaining solution. If the NE criteria are not satisfied for energy allocation implied by the bargaining solution, then both nodes will use direct transmission in the current stage game.

\section{IV. $K>\mathbf{2}$ PLAYER RELAYING GAME}

The previous section establishes how a pair of selfish source nodes can arrive at a mutually agreeable energy allocation through bargaining and also establishes the conditions under which this pair of nodes will not defect from their bargaining agreement. This section extends these results to transmission sessions with $K>2$ source nodes. We restrict our attention here to the particular scenario in which the source nodes form fixed two-player partnerships for the duration of a transmission session. While this restriction excludes more general network configurations, e.g., relaying coalitions with more than two nodes or dynamic partnerships, the restriction to fixed partnerships is based on the principle that nodes are less likely to defect if the probability of additional interactions with their current partner is high; dynamic partnerships among $K$ selfish source nodes when $K$ is large can result in non-cooperative behavior because of the low likelihood of additional interactions after the current frame. The central problem is then the assignment of partners to each source node (note that one source will have no partner when $K$ is odd). Specifically, we consider the problem of how to form stable partnerships endogenously by a network of selfish source nodes.

We define a pairing instance $\mathcal{P}$ as a set of two-player partnerships in which all but at most one source nodes are disjointly paired. It is not difficult to show that each pairing instance in a $K>2$ player relaying game is an equilibrium with respect to unilateral deviations when the energy allocations are determined by a bargaining solution and the NE conditions are satisfied. Pairing instances may not be an equilibrium with respect to multi-player deviation, however, where two or more players leave their current partners and form different partnerships. As an example, consider a network with $K=4$ source nodes denoted as $\mathcal{S}=$ $\{1,2,3,4\}$ and a pairing instance $\mathcal{P}=\{\{1,2\},\{3,4\}\}$. Suppose that all nodes receive an identical expected payoff of $\pi_{\mathcal{P}}>$ 0 under this pairing instance. Suppose further that, under pairing instance $\mathcal{Q}=\{\{1,3\},\{2,4\}\}$, nodes 1 and 3 each receive a payoff of $\pi_{\mathcal{Q}}>\pi_{\mathcal{P}}$ while nodes 2 and 4 receive a payoff of zero. It is clear that nodes 1 and 2 both improve their payoff by deviating from pairing instance $\mathcal{P}$ to $\mathcal{Q}$, and they can do so without any consent (or repercussions) from nodes 3 and 4 . Hence, although pairing instance $\mathcal{P}$ is an equilibrium with respect to unilateral deviation, it is not an equilibrium with respect to multi-player deviations.

While there are many notions of stability in $K>2$ player games, we restrict our attention here to the notion of a pairwisestable network [17]. A pairwise-stable network is a pairing instance that is immune to any improving two-player deviations, where an improving two-player deviation in our context is a deviation in which two players sever their current partnerships and form a new partnership such that at least one player in the new partnership receives a strictly greater expected payoff while the other player in the new partnership receives an expected payoff no worse than before. Pairing instance $\mathcal{P}$ in the previous paragraph is clearly not pairwise stable.

The problem of how to endogenously form a pairwise-stable network among selfish nodes has been studied extensively under the title of stable matching problems [11]. Stable matching problems are generally divided into two categories: Twosided matching and one-sided matching. In a two-sided matching problem, also referred to as the marriage problem, there are two disjoint sets of participants and the matching is a one-toone mapping between the two sets. This is the classic matching problem discussed in [11], where it is shown that every instance of the two-sided matching problem always admits at least one pairwise-stable solution. In the one-sided matching problem, a matching results in a partition of the single set of participants into disjoint pairs. This is a generalization of the marriage problem and is known as the roommate problem. A major difference between a two-sided (marriage) problem and a one-sided (roommate) problem is that the roommate problem may not necessarily have a stable matching.

In our context with $K>2$ source nodes that need to endogenously form a pairing instance to receive positive payoffs, the matching problem is one-sided since the source nodes are homogeneous. In the absence of central control, the source nodes can attempt to form a stable matching at the start of the transmission session by first computing the expected bargaining payoffs (also taking into consideration that the bargaining payoff is zero if the energy allocation does not satisfy the NE conditions) for each of the $K-1$ possible partners in the network. These payoffs then imply a preference table, known to each node, that is used to determine a pairwise-stable matching, if one exists. If a pairwise-stable matching exists, the nodes then cooperate in the transmission session with these pairings using transmission/relaying energies specified by the appropriate bargaining solution (or direct transmission if the bargaining solution specifies disagreement or does not satisfy the $\mathrm{NE}$ conditions). If a pairwise-stable matching does not exist, one approach is to resort to direct transmission in the current transmission session. Another approach is to locally compute the centrally controlled pairings, i.e., the pairing instance that would result in the maximum sum (or product) payoff over all of the source nodes, and form partnerships based on this pairing. We note that both of these solutions are stable with respect to unilateral deviations 
Table 1. Preference table for a 4-source matching problem.

\begin{tabular}{c|ccc}
\hline \hline Source & $\begin{array}{c}\text { First } \\
\text { preference }\end{array}$ & $\begin{array}{c}\text { Second } \\
\text { preference }\end{array}$ & $\begin{array}{c}\text { Third } \\
\text { preference }\end{array}$ \\
\hline 1 & 4 & 2 & 3 \\
2 & 1 & 3 & 4 \\
3 & 2 & 4 & 1 \\
4 & 3 & 1 & 2 \\
\hline
\end{tabular}

but may not be pairwise stable.

As an example of finding stable matchings, consider a scenario in which $K=4$ sources compute their bargaining solutions with each other and generate the preference table given in Table 1. In this example, it is impossible for each node to be paired with their first preference since, for example, node 1 prefers node 4 , but node 4 prefers node 3 . Nevertheless, it is not difficult to verify that $\{(1,2),(3,4)\}$ is a stable matching for this example. Source 1 would prefer source 4 to source 2 , but source 4 is paired with his first choice and is unwilling to deviate from this solution. Similarly, source 3 prefers source 2 to source 4 , but source 2 is paired with his first choice. Of the other possible matchings, it is not difficult to show that $\{(1,4),(2,3)\}$ is stable and $\{(1,3),(2,4)\}$ is unstable. For small networks, it is straightforward to simply test all possible matchings for stability. For larger networks, a more efficient algorithm for finding stable matchings with complexity of $O\left(n^{2}\right)$ for systems with $2 n$ players is provided in [18].

\section{NUMERICAL RESULTS}

To compare the performance of wireless networks with selfish energy allocation and endogenous partner selection to that of networks under central control, this section provides numerical examples demonstrating the relative energy efficiency of several schemes under two performance metrics: (i) The total energy savings of the network (sum payoff) and (ii) the product of the energy savings of the network (product payoff), both with respect to direct transmission. In all of the numerical results presented here, we assume a wireless network using OAF cooperative transmission, a discount factor of $\delta=0.98$, and an SNR $=10 \mathrm{~dB}$ QoS requirement. In each transmission session, $K$ source and $K$ destination nodes are randomly placed (with uniform distribution) on a disk of radius $R=10$ meters. The relative energy efficiency of several schemes, averaged over the random node positions (and over the channel realizations in systems with fading channels), are compared to a system with centrally controlled (CC) energy allocations, i.e., the energy allocation resulting in the maximum sum/product payoff for each pair, and $\mathrm{CC}$ pairing assignments, i.e., the pairing assignment resulting in the maximum sum/product payoff over the network. A relative energy efficiency of one corresponds to the maximum network payoff according to the sum/product payoff metric (obtained through $\mathrm{CC}$ energy allocations and $\mathrm{CC}$ pairing assignments) and a relative energy efficiency of zero corresponds to direct transmission.

Fig. 5 shows the relative energy efficiency of a wireless network using cooperative transmission through non-fading pathloss channels under the sum payoff metric. The squared chan-

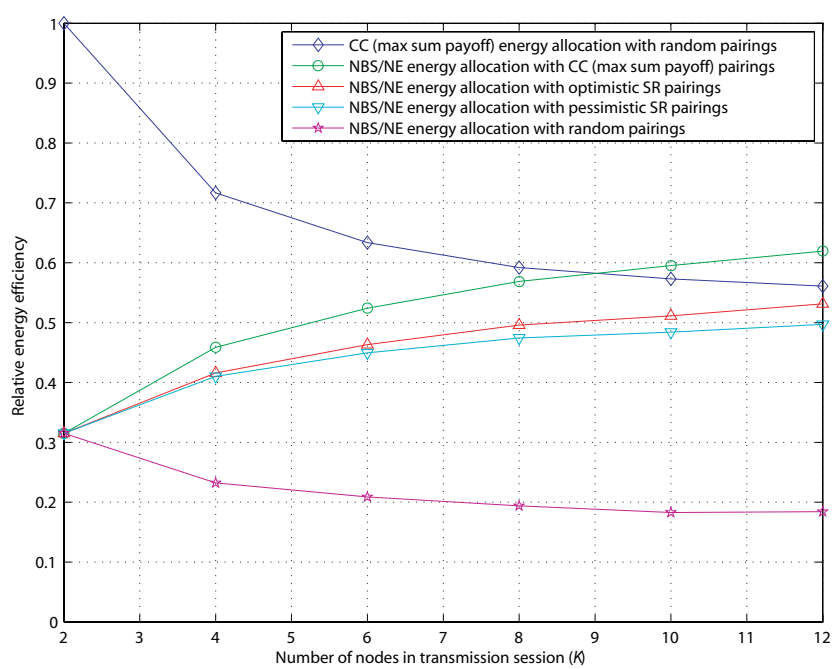

Fig. 5. Relative energy efficiency of a wireless network under the sum payoff metric with non-fading pathloss channels using OAF cooperative transmission with $\mathrm{CC}$ or selfish (NBS/NE) energy allocation and with random, CC, or optimistic/pessimistic SR pairing assignments.

nel magnitude between each node pair $i$ and $j$ is calculated as $H_{i j}=\left(\left(x_{i}-x_{j}\right)^{2}+\left(y_{i}-y_{j}\right)^{2}\right)^{-\gamma / 2}$ where $\gamma=4$ is the pathloss parameter and $\left(x_{i}, y_{i}\right)$ is the cartesian coordinate pair of node $i$. The $\mathrm{CC}$ energy allocations in this case correspond to the maximum sum payoff for each node pair and the CC pairing assignments correspond to the maximum sum payoff over the network. These results show that a system with purely selfish energy allocations (NBS/NE) and endogenously formed node pairings (SR) can achieve a relative energy efficiency of approximately half of that of a system with CC energy allocations and CC pairings for values of $K \geq 10$. Since a stable roommate node pairing solution does not always exist, Fig. 5 shows "optimistic" and "pessimistic" bounds on the relative energy efficiency of NBS/NE energy allocations with SR pairings by using the CC pairing assignment and direct transmission, respectively, when a SR pairing solution is not found. Note that the results corresponding to CC energy allocations are not stable with respect to unilateral deviations because some nodes may receive negative payoffs under a CC energy allocation and these nodes would rationally choose defection. Also note that all of the results corresponding to NBS/NE energy allocations are stable with respect to unilateral deviations in the sense that no single node can improve its payoff by defecting. The NBS/NE results with SR pairings are pairwise stable when the SR pairing solution exists, whereas the $\mathrm{CC}$ and random pairings are not.

Fig. 6 shows the relative energy efficiency of a wireless network using cooperative transmission through non-fading pathloss channels under the product payoff metric. As discussed in [19] and [20], the product payoff metric, unlike sum payoff, accounts for some degree of fairness in the payoffs since no node should be given a small (or zero) payoff when the goal is to maximize the product payoff. The CC energy allocations in this case correspond to the NBS energy allocations for each node pair (since, according to its definition in (1), the NBS maximizes the product payoff for a given node pair) and the $\mathrm{CC}$ pairing assignments correspond to the maximum product payoff over the 


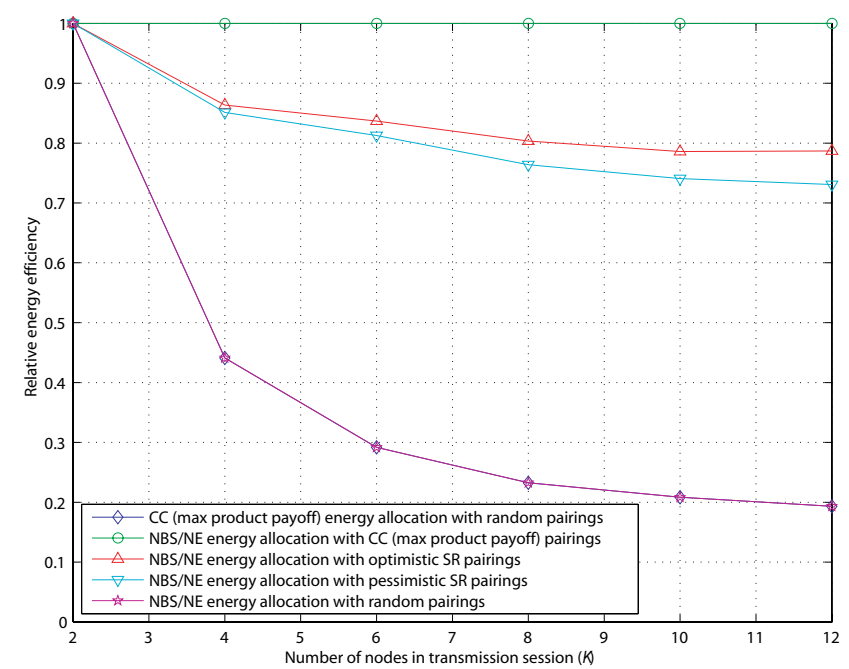

Fig. 6. Relative energy efficiency of a wireless network under the product payoff metric with non-fading pathloss channels using OAF cooperative transmission with $\mathrm{CC}$ or selfish (NBS/NE) energy allocation and with random, CC, or optimistic/pessimistic SR pairing assignments.

network. Since the CC energy allocations are the NBS energy allocations in this example, this metric also allows us to isolate the effect of the pairing mechanism on the efficiency of the network. The results in Fig. 6 show that the SR pairing scheme can provide more than $75 \%$ of the product payoff of the CC pairing scheme in terms of energy efficiency for the network, and clearly outperforms random pairing by margin of $50 \%$ at $N=10$ nodes. Note that when we implement NBS locally on a per node basis, the nodes cooperate only if the $\mathrm{NE}$ criterion is satisfied. However, when we use the NBS energy allocations in central manner, the nodes are forced to cooperate regardless of the NE criterion. The results in Fig. 6 demonstrate that satisfying NE criteria has an almost negligible effect on the energy efficiency of the network in non-fading channels.

Fig. 7 shows the relative energy efficiency of a wireless network using cooperative transmission through quasi-static fading channels under the sum payoff metric. The channel means are specified according to the path-loss model used in the previous simulations and the channel realizations $h_{i j}[n]$ are generated independently (both spatially and temporally) according to the Nakagami- $m$ distribution with $m=2$. The results in this case are similar to those in Fig. 5, with all of the selfish techniques performing slightly better in fading channels with respect to a system under central control. A system with purely selfish energy allocations (NBS/NE) and endogenously formed node pairings (pessimistic SR) can achieve an energy efficiency of better than $50 \%$ of that of a system with CC energy allocation and CC pairings for $N \geq 6$.

Overall, in the absence of central control in a wireless ad hoc network, these numerical examples confirm that there is a price to pay for selfish behavior in terms of the overall energy efficiency of the network. Whether this price is too high depends to a large extent on the application. Nevertheless, these results also demonstrate that a network with sources that endogenously form cooperative pairs and selfishly allocate transmission/relaying energies without any external incentive mecha-

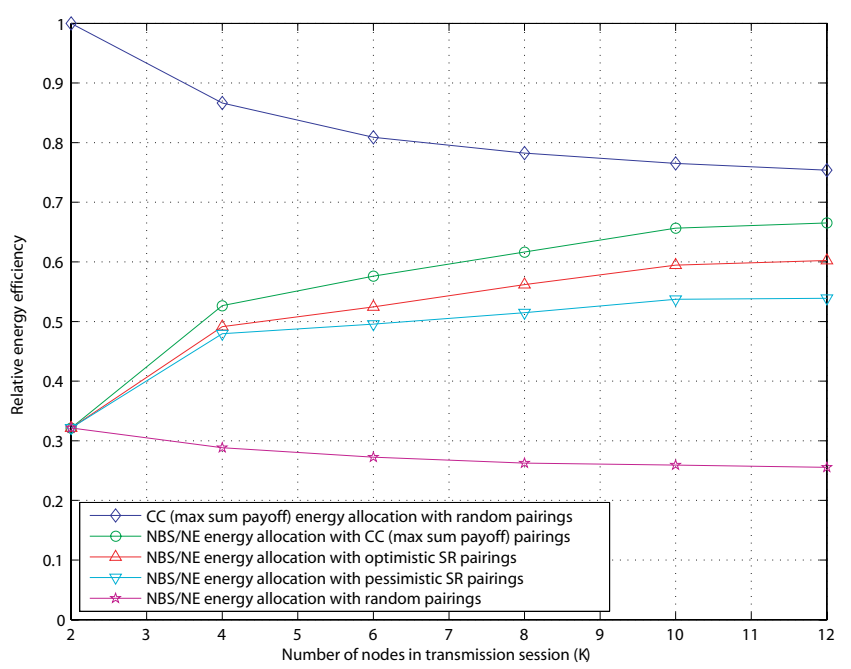

Fig. 7. Relative energy efficiency of a wireless network under the sum payoff metric with Nakagami- $m(m=2)$ independent fading channels using OAF cooperative transmission with CC or selfish (NBS/NE) energy allocation and with random, CC, or optimistic/pessimistic SR pairing assignments.

nisms or altruistic nodes to enforce cooperation can significantly improve the overall energy efficiency of the network with respect to that of direct transmission.

\section{CONCLUSION}

This paper employs both cooperative and non-cooperative game theoretic tools to analyze the energy efficiency of wireless ad hoc networks with selfish energy allocation and endogenous partner selection. The novelty of this study is that cooperation is established without the added complexity of extrinsic incentive mechanisms, altruistic nodes, and/or community enforcement. We first used axiomatic bargaining tools from cooperative game theory to describe how two selfish source nodes can decide on a mutually-agreeable Pareto-efficient energy allocation that can be locally computed without central authority in scenarios where mutual benefit is possible through cooperative transmission. We then analyzed the conditions under which selfish nodes would follow through on this agreement and not defect in a repeated relaying game scenario and developed the necessary and sufficient conditions under which "natural" cooperation is possible for both fading and non-fading channels. We then extended the two-player model to networks with $K>2$ players and proposed a technique to endogenously form cooperative partnerships without central control through the stable roommate algorithm. Finally, we provided numerical results based on OAF cooperative transmission to quantify the energy efficiency of an ad hoc wireless network with selfish sources with respect to a network with centrally optimized energy allocations and pairings.

\section{APPENDIX}

This appendix describes a set of sufficient conditions under which the non-negative feasible payoff set $\mathcal{U}$ is convex, closed, and bounded from above. Recall that the payoff received by node $j$ is defined as the energy saved by node $j$ with respect 
to direct transmission. When node $i \neq j$ is the relay for node $j$, this payoff can be expressed as

$$
\pi_{j}=\mathcal{E}_{j}^{*}-\mathcal{E}_{j}^{r_{\text {min }}}=\psi_{j}\left(\mathcal{E}_{i}^{r_{\text {min }}}\right)-\mathcal{E}_{j}^{r_{\text {min }}}
$$

where $\psi_{j}:[0, \infty) \mapsto\left[0, \mathcal{E}_{j}^{d t}\right)$ is the relay energy function that maps the relaying energy offered by node $i$, i.e., $\mathcal{E}_{i}^{r_{\text {min }}}$, to the reduction in transmit energy with respect to direct transmission at node $j$, i.e., $\mathcal{E}_{j}^{*}$. Note that $\psi_{j}$ is bounded from above since it is impossible for node $j$ to reduce its transmit energy by more than $\mathcal{E}_{j}^{d t}$. It is also reasonable to assume that any useful relay energy function $\psi_{j}$ is monotonically increasing since any increase in the relaying energy offered by node $i$ should not diminish the reduction in transmit energy experienced by node $j$. In addition to these properties, we assume that $\psi_{j}$ is concave and continuous. The concavity condition, in particular, is intuitively justified by the fact that each increase of the relaying energy offered by node $i$ should result in smaller reductions in transmit energy at node $j$. We note that the amplify-and-forward relaying function satisfies all of these properties [12].

Before proving that a pair of nodes using monotonically increasing, concave, continuous, and bounded from above relay energy functions have a non-negative feasible payoff set that is convex, closed, and bounded from above, we will first show that if $\left(\pi_{i}, \pi_{j}\right)$ is a feasible payoff pair, then all payoff pairs $\left(\pi_{i}^{\prime}, \pi_{j}^{\prime}\right)$ such that $\pi_{i}^{\prime} \leq \pi_{i}$ and $\pi_{j}^{\prime} \leq \pi_{j}$ are also feasible.

Lemma 1: Assume $\psi_{i}$ and $\psi_{j}$ are monotonically increasing, concave, continuous, and bounded from above. If $\left(\pi_{i}, \pi_{j}\right)$ is a feasible payoff pair, then any payoff pair $\left(\pi_{i}^{\prime}, \pi_{j}^{\prime}\right)$ such that $\pi_{i}^{\prime} \leq \pi_{i}$ and $\pi_{j}^{\prime} \leq \pi_{j}$ is also feasible.

Proof: The proof follows from the fact that either node can reduce its payoff arbitrarily by increasing its relay energy. Each node's relaying energy is continuous and unbounded. Suppose node $i$ increases its relaying energy to reduce its payoff to $\pi_{i}^{\prime}$. Since $\psi_{j}$ is monotonically increasing, this increase in relaying energy by node $i$ may lead to an increased payoff at node $j$. Hence, node $j$ must increase its relaying energy energy to reduce its payoff to $\pi_{j}^{\prime}$. This, in turn, may lead to an increased payoff at node $i$, hence node $i$ must again increase its relaying energy to reduce its payoff to $\pi_{i}^{\prime}$. This iteration is repeated between nodes $i$ and $j$ and converges because $\psi_{i}$ and $\psi_{j}$ are concave, continuous, and bounded from above. Hence, it is possible for both nodes achieve any payoff pair $\left(\pi_{i}^{\prime}, \pi_{j}^{\prime}\right)$ with $\pi_{i}^{\prime} \leq \pi_{i}$ and $\pi_{j}^{\prime} \leq \pi_{j}$

This result will be used in the following lemma.

Lemma 2: If $\psi_{i}$ and $\psi_{j}$ are monotonically increasing, concave, continuous, and bounded from above then the nonnegative feasible payoff set $\mathcal{U}$ is convex, closed, and bounded from above.

Proof: We will first prove that $\mathcal{U}$ is convex. For notational convenience, let $\left(x_{i}, x_{j}\right):=\left(\mathcal{E}_{i}^{r_{\min }}, \mathcal{E}_{j}^{r_{\min }}\right)$ denote a valid relaying energy allocation with $x_{i} \geq 0$ and $x_{j} \geq 0$. Suppose there exists two feasible payoff pairs in $\mathcal{U}$ with valid relaying energy allocations denoted as $\left(x_{i}, x_{j}\right) \rightarrow\left(\pi_{i}, \pi_{j}\right)$ and $\left(x_{i}^{\prime}, x_{j}^{\prime}\right) \rightarrow$ $\left(\pi_{i}^{\prime}, \pi_{j}^{\prime}\right)$. We will show that $\left(\alpha \pi_{i}+(1-\alpha) \pi_{i}^{\prime}, \alpha \pi_{j}+(1-\alpha) \pi_{j}^{\prime}\right)$ is also in $\mathcal{U}$ for all $0 \leq \alpha \leq 1$, hence the $\operatorname{set} \mathcal{U}$ is convex.

Form the relaying energy allocation $\left(x_{i}^{\prime \prime}, x_{j}^{\prime \prime}\right)=\alpha\left(x_{i}, x_{j}\right)+$ $(1-\alpha)\left(x_{i}^{\prime}, x_{j}^{\prime}\right)$. Note that this relaying energy allocation is valid for all $0 \leq \alpha \leq 1$. The payoff for node $i$ is

$$
\begin{aligned}
\pi_{i}^{\prime \prime} & =\psi_{i}\left(\alpha x_{j}+(1-\alpha) x_{j}^{\prime}\right)-\alpha x_{i}-(1-\alpha) x_{i}^{\prime} \\
& \geq \alpha \psi_{i}\left(x_{j}\right)+(1-\alpha) \psi_{i}\left(x_{j}^{\prime}\right)-\alpha x_{i}-(1-\alpha) x_{i}^{\prime} \\
& =\alpha \pi_{i}+(1-\alpha) \pi_{i}^{\prime}
\end{aligned}
$$

where the inequality is a consequence of the concavity of $\pi_{i}$. Similarly, $\pi_{j}^{\prime \prime} \geq \alpha \pi_{j}+(1-\alpha) \pi_{j}^{\prime}$. Hence, if $\left(\pi_{i}, \pi_{j}\right)$ and $\left(\pi_{i}^{\prime}, \pi_{j}^{\prime}\right)$ are feasible payoff pairs and $0 \leq \alpha \leq 1$, the nodes can achieve another feasible payoff pair $\left(x_{i}^{\prime \prime}, x_{j}^{\prime \prime}\right) \rightarrow\left(\pi_{i}^{\prime \prime}, \pi_{j}^{\prime \prime}\right)$ with a valid relaying energy allocation such that $\pi_{i}^{\prime \prime} \geq \alpha \pi_{i}+(1-\alpha) \pi_{i}^{\prime}$ and $\pi_{j}^{\prime \prime} \geq \alpha \pi_{j}+(1-\alpha) \pi_{j}^{\prime}$. Lemma 1 implies that the payoff pair $\left(\alpha \pi_{i}+(1-\alpha) \pi_{i}^{\prime}, \alpha \pi_{j}+(1-\alpha) \pi_{j}^{\prime}\right)$ must also be feasible, hence $\mathcal{U}$ is convex.

To see that $\mathcal{U}$ is closed and bounded from above, let $\mathcal{X}=$ $\left\{\left(x_{i}, x_{j}\right): 0 \leq x_{i} \leq \mathcal{E}_{i}^{d t}\right.$ and $\left.0 \leq x_{j} \leq \mathcal{E}_{j}^{d t}\right\}$. Note that $\mathcal{X}$ is a compact set and includes all of the relaying energy allocations under which both nodes receive a non-negative payoff. Let $\mathcal{Y}=$ $\left\{\left(\pi_{i}, \pi_{j}\right):\left(x_{i}, x_{j}\right) \rightarrow\left(\pi_{i}, \pi_{j}\right) \forall\left(x_{i}, x_{j}\right) \in \mathcal{X}\right\}$ be the image of $\mathcal{X}$ in the payoff plane. Note that $\mathcal{U} \subseteq \mathcal{Y}$ and that $\mathcal{Y}$ must also be compact since $\psi_{i}$ and $\psi_{j}$ are continuous functions. The set $\mathcal{U}$ can be formed by taking the intersection of $\mathcal{Y}$ with the set $\mathcal{Z}=\left\{\left(\pi_{i}, \pi_{j}\right): 0 \leq \pi_{i} \leq \mathcal{E}_{i}^{d t}\right.$ and $\left.0 \leq \pi_{j} \leq \mathcal{E}_{j}^{d t}\right\}$. Since the intersection of two compact sets is also compact, $\mathcal{U}$ must also be compact. Hence, $\mathcal{U}$ is closed and bounded from above.

\section{REFERENCES}

[1] Q. Zhao and L. Tong, "Energy efficiency of large-scale wireless networks: Pro-active vs. reactive networking," IEEE J. Sel. Areas Commun., vol. 23, no. 5, pp. 1100-1112, May 2005.

[2] S. Marti, T. J. Giuli, K. Lai, and M. Baker, "Mitigating routing misbehavior in mobile ad hoc networks," in Proc. ACM/IEEE Mobicom, 2000, pp. 255-265.

[3] J. Yang, A. G. Klein, and D. Brown III, "Cooperation without extrinsic incentive mechanisms in wireless networks with selfish nodes," to be appeared in IEEE Signal Process. Mag., 2009.

[4] J.-P. Hubaux, T. Gross, J.-Y. Le Boudec, and M. Vetterli, "Towards selforganized mobile ad hoc networks: The terminodes project," IEEE Commun. Mag., vol. 39, no. 1, pp. 118-124, Jan. 2001.

[5] L. Blazevic, L. Buttyan, S. Capkun, S. Giordano, J.-P. Hubaux, and J.-Y. L. Boudec, "Self organization in mobile ad hoc networks: The approach of terminodes," IEEE Commun. Mag., vol. 39, no. 6, pp. 166-174, June 2001.

[6] M. Jakobsson, J.-P. Hubaux, and L. Buttyan, "A micro-payment scheme encouraging collaboration in multi-hop cellular networks," Financial Crypto, 2003.

[7] L. Lai and H. El Gamal, "On cooperation in energy efficient wireless networks: The role of altruistic nodes," IEEE Trans. Wireless Commun., vol. 7, no. 5, pp. 1868-1878, May 2008.

[8] M. Felegyhazi, J.-P. Hubaux, and L. Buttyan, "Nash equilibria of packet forwarding strategies in wireless ad hoc networks," IEEE Trans. Mobile Comput., vol. 5, pp. 1044-1049, May. 2006.

[9] J. Yang and D. R. Brown III, "Energy efficient relaying games in cooperative wireless transmission systems," in Proc. the Forty-First Asilomar Conference on Signals, Systems and Computers, Nov. 2007, pp. 835-839.

[10] J. N. Laneman, D. N. C. Tse, and G. W. Wornell, "Cooperative diversity in wireless networks: Efficient protocols and outage behavior," IEEE Trans. Inf. Theory, vol. 50, no. 12, pp. 3062-3080, 2004.

[11] D. Gale and L. Shapley, "College admissions and the stability of marriage," The American Mathematical Monthly, vol. 69, no. 1, pp. 9-15, Jan. 1962.

[12] J. Yang, D. Gündüz, D.R. Brown III, and E. Erkip, "Resource allocation for cooperative relaying," in Proc. CISS, Princeton, NJ, Mar 2008.

[13] K. Binmore, Fun and Games: A Text on Game Theory. Lexington, MA: D.C. Heath, 1992.

[14] E. Kalai and M. Smorodinsky, "Other solutions to Nash's bargaining problem," Econometrica, vol. 43, no. 3, pp. 513-518, 1975.

[15] X. Cao, "Preference functions and bargaining solutions," in Proc. IEEE Conf. Decision and Control, 1982, pp. 164-171. 
[16] R. Axelrod, The Evolution of Cooperation. Basic Books, 1984.

[17] M. Jackson and A. Wolinsky, "A strategic model of social and economic networks," Journal of Economic Theory, vol. 71, no. 1, pp. 44-74, June 1996.

[18] D. Gusfield and R. W. Irving, The Stable Marriage Problem: Structure and Algorithms. Cambridge, MA: MIT Press, 1989.

[19] Y. Chen, P. Cheng, P. Qiu, and Z. Zhang, "Optimal partner selection strategies in wireless cooperative networks with fixed and variable transmit powers," in Proc IEEE WCNC, Mar. 2007, pp. 4080-4084.

[20] R. Mazumdar, L. G. Mason, and C. Douligeris, "Fairness in network optimal flow control: Optimality of product forms," IEEE Trans. Commun. vol. 39, no. 5, pp. 775-782, May 1991.

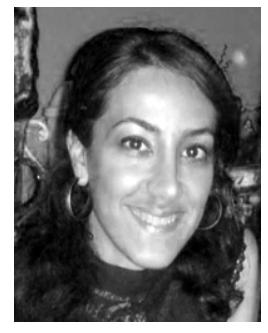

Fatemeh Fazel is a Postdoctoral Associate at Northeastern University. She received a Ph.D. in Electrical Engineering and Computer Science from the University of California in Irvine in 2008, an M.Sc. degree from the University of Southern California in 2002, and the B.Sc. degree from Sharif University of Technology in 2000 .

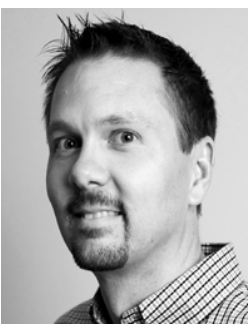

D. Richard Brown III is an Associate Professor in the Department of Electrical and Computer Engineering at Worcester Polytechnic Institute. He received a Ph.D. in Electrical Engineering from Cornell University in 2000 and M.S. and B.S. degrees in Electrical Engineering from The University of Connecticut in 1996 and 1992, respectively. He is a Senior Member of the IEEE and also held an appointment as a Visiting Associate Professor at Princeton University from August 2007 to June 2008. 